\title{
O ENSINO DA ANÁLISE DE ASSUNTO: em busca de uma metodologia
}

\section{THE TEACHING OF SUBJECT ANALYSIS: in search of a methodology}

\author{
Gercina Ângela de Lima'
}

\section{RESUMO}

A indexação é um dos processos mais importantes em SRI para garantir a recuperação da informação e o acesso a ela, mas nem sempre o indexador possui uma formação especializada na área de tratamento de conteúdos de documentos, tanto do ponto de vista técnico quanto do ponto de vista teórico. Sendo assim, é um processo que deve ser ensinado e aprendido pelos bibliotecários em todos os seus aspectos, por ser uma formação especializada, no âmbito do curso de biblioteconomia. Este artigo teve como objetivo lançar um olhar sobre a literatura existente com o intuito de conhecer o que de fato tem sido pesquisado sobre o ensino da Análise de assunto, para melhorar a subjetividade desse processo e contribuir para o ensino e a aprendizagem dessa disciplina, no âmbito dos cursos de graduação em Biblioteconomia. Estapesquisaenvolveuprocedimentos denaturezateórica,empírica, descritivaeaplicada,comabordagemqualitativaem relação à análise da literatura especializada da área, com resultados parciais de reflexões realizadas no âmbito do projeto de pesquisa PQ-CNPq. Constatou-se que existem muitas lacunas a serem resolvidas em relação ao ensino da Análise de assunto pelo seu caráter subjetivo, e que, por conseguinte, requer do aluno raciocínios lógicos e cognitivos, além de conhecimentodalinguística edatipologiadocumental.Ficouevidenteanecessidadedeseterumcursobemestruturado, compostodetemáticasquepossibilitemoaprendizadoeodesenvolvimentodehabilidadesnecessáriasparaarealizaçãoda atividade de análise de assunto, tendo um equilíbrio entre a teoria e a prática.

Palavras-chave: Ensino da Análise de Assunto. Ensino da Indexação. Metodologias. Análise de Assunto. Indexação.

\section{ABSTRACT}

Indexing is one of the mostimportant processes in SRI toguaranteetheretrieval and access to information, buttheindexer does notalways have specialized training in the area of handling document content, both from atechnical and theoretical point of view. Therefore, it is a process that must be taught and learned by librarians in all its aspects, as it is a specialized training, within the scope of the library science course. This article aimed to take a look at the existing literature in order to know whathas actually been researchedaboutteaching subjectanalysis, to improvethesubjectivity of this process and contribute to the teaching and learning of subject analysis, within the scope of undergraduate courses in Library Science. This research involved theoretical,empirical, descriptiveandapplied procedures, with aqualitativeapproach in relationto the analysis of the specialized literature in the area, with partial results of reflections carried out within the scope of the PQ-CNPq research project.It was found that there are many gaps to be solved in relation to the teaching of subjectanalysis duetoitssubjectivecharacter, and that, therefore, requireslogical and cognitivereasoning from thestudent, inadditionto knowledgeoflinguisticsanddocumentarytypology.Itbecameevidenttheneedtohaveawell-structuredcourse,composed of themes that enable learning, and the development of skills necessary to carry out the subject analysis activity, having a balance between theory and practice.

Keywords: Teaching Subject Analysis. Teaching of Indexing. Methodologies. Subject Analysis. Indexing.

\footnotetext{
1 Professora Titular na Escola de Ciência da Informação. Universidade Federal de Minas Gerais, Brasil ORCID http://orcid.org/0000-0003-0735-3856. E-mail: glima@eci.ufmg.br
} 


\section{INTRODUÇÃO}

Aindexação éum importante processo dentro de um Sistema de Recuperação da Informação - SRI, composto das etapas de análise de assunto e tradução. A primeira etapa consiste em analisaro documento,identificarconceitosqueorepresentemeselecionaraquelesquesejammaisadequadosà concepçãoadotadapeloindexador-simplista,orientadaparaoconteúdo,orientadapelademanda,ou centradanousuário.Nasegundaetapa,oindexadorutilizadelinguagensdeindexação paratraduziros termos selecionados, de modo a facilitar a recuperação pelo usuário do SRI das informações contidas neles.

Sabe-se que localizar informações por assunto é uma das abordagens fundamentais a todos os que procuram informações. Para aprimorar a recuperação da informação nos SRIs, o processo de indexação precisa de profissionais com conhecimentos teóricos e práticos de organização da informação. Algumas profissões, muitas vezes, desvalorizam suas características práticas, como é o caso da profissão dos bibliotecários que possuem várias funções de serviços técnicos; no entanto, éimportante reconhecer a indexação como uma habilidade importante de ser ensinada eaprendida pelos bibliotecáriosemtodososaspectos docurso debiblioteconomia.Naliteraturadaárea, aolongo dos anos, nota-se um debate que discute se os conteúdos das disciplinas dos currículos do curso de Biblioteconomia devem concentrar-se na teoria ou na prática. Porém, este presente estudo parte do pressuposto de que ambas as abordagens devem ser ensinadas - nem a teoria sem as habilidades práticas, nem as habilidades práticas sem uma compreensão fundamentada da teoria.

Assim, para trabalhar como indexador, é necessário ter uma formação especializada na área detratamentodeconteúdos dedocumentos, tanto do pontodevistatécnico quantodoteórico. Além disso, as experiências e os conhecimentos prévios desse profissional diferenciarão o resultado do tratamento informacional realizado por outro indexador menos preparado. Naves $(2004$, p. 8$)$ afirma que:

Oprofissionaldainformaçãoquedesenvolveaatividadedeindexarassuntosdedocumentos é chamado de indexador, catalogador de assuntos ou classificador. A maioria desses profissionais é graduada em Biblioteconomia, e deve conhecer os fundamentos teóricos e técnicos do tratamento temático da informação (NAVES, 2004, p. 8).

Com esses pontos em mente, é razoável afirmar que todos os bibliotecários devem ter uma compreensão básica dos princípios da área de organização da informação, e também devem possuir habilidades básicas para realizarem o processo de indexação, tanto nos procedimentos da primeira 
etapa, a análise deassunto, quanto nos procedimentos da segunda etapa, a tradução, na qual ocorrea representação da informação por meio do uso dos vocabulários controlados.

Para isso, é papel dos educadores, com responsabilidade para com a profissão, garantir aos alunos a aprendizagem de"como"fazer, mastambémo"porque"fazer, tendo um equilíbrioentre uma abordagempráticafundamentadanateoria.Noentanto,osautores Mulvany(1994)eAnderson(2002) concordamqueaatividadeanálisedeassuntopersi só, não podeserensinada, porenvolverprocessos cognitivoscomplexosnaleituraenainterpretaçãododocumentoena seleçãodostermosque melhor representemseuconteúdo.Assim, acapacidadedeensinareanalisarotextoobjetivamenteapartirde sua estrutura textual pode ser orientada, mas difícil de ser ensinada.

Constatando-seaexistênciadecarênciadeliteraturasque proporcionem diretrizes explícitas paraoensinodesseprocessotãosubjetivoeapequenaquantidadedepesquisasteóricasdesenvolvidas sobre metodologia e ensino, este estudo objetiva lançar um olhar sobre a literatura existente e sobre as reflexões ocorridas no âmbito da sala de aula, com o intuito de conhecer o que de fato tem sido pesquisado sobre o ensino dessa temática e propor melhorias.

Nesse contexto, foram feitos os seguintes questionamentos para direcionar este estudo: (1) é possível melhorar o ensino do processo de análise de assunto?; (2) quais são as habilidades necessárias para ensinar, compreender e aprender este processo?; e, finalmente, (3) quais são as responsabilidades do professor no ensino e do aluno na aprendizagem?

Somado a isso, conta a experiência da autora deste trabalho de mais de 15 anos em lecionar a disciplina Análise de assunto para os alunos do curso de Biblioteconomia da Escola de Ciência da Informação da UFMG, ea pesquisa sobre metodologia deensino de Análise de assunto, queéo objeto de pesquisa do projeto da autora em andamento"Estudo sobre o estatuto teórico metodológico da análise de assunto", no âmbito do projeto de pesquisa PQ-CNPq, o qual tem como objetivo estudar os fundamentos teóricos metodológicos da análise de assunto, verificando, principalmente, a evolução metodológica e as contribuições de pesquisas que visam melhorar a subjetividade desse processoe seu ensino na graduação. Além disso, a sala de aula tornou-se um laboratório experimental no qual a autora vem testando abordagens de ensino do ponto de vista cognitivo, com os aspectos linguísticos e lógicos.

Portanto, este artigo apresenta os resultados parciais de reflexões realizadas sobre o ensino e a aprendizagem da disciplina Análise de assunto no âmbito desse projeto de pesquisa e a partir de experiências vivenciadas em sala em aula. Nas próximas seções, apresenta-se, primeiramente, a 
metodologia,seguidadeumestudodavariaçãoterminológicadacompreensãodoconceitodeassunto; depois discorre-se sobre o panorama do processo de análise de assunto; em seguida, são feitas reflexões a partir da literatura sobre o ensino e a aprendizagem dessa temática; e as considerações finais.

\section{METODOLOGIA}

Em uma pesquisa, é fundamental o conhecimento de outros trabalhos que tenham sido realizados sobrea temática específica a serestudada para que se possa conhecere mapearo estado da arte de seu objeto de estudo. Portanto, a revisão de literatura se caracteriza por trazer à luz, de forma norteadora e reflexiva, trabalhos que reforçam ou confirmam os pressupostos inicialmente previstos na pesquisa. No caso deste artigo, será realizada uma revisão narrativa de literatura, já que a pesquisa é conduzida por questões mais abertas, com o intuito de conhecer o estado da arte sobre o ensino da disciplina Análise de assunto, mas especificamente para verificar metodologias de ensino eaprendizagem desse processo que possui uma alta carga subjetiva na compreensão do conteúdo de um documento.

Esta pesquisa, como um todo, tem uma abordagem quali-quantitativa, pois, no seu decorrer, seráusadaaanálisedosdadosquantitativamente;éuma pesquisa exploratóriaedescritiva, poispartese de um levantamento bibliográfico, buscando analisar e descrever os resultados. Para estudar o objeto da pesquisa e atingir os objetivos, propôs-se, primeiramente, elaborar uma revisão narrativa da literatura, para: (1) mapear a literatura sobre o objeto estudado e (2) coletar insumos iniciais para embasarapropostateórico-metodológicanestaetapadapesquisa.Essescritériossejustificamporque a análise deassuntoéutilizada, algumasvezes, para referir-seà primeira etapa;outrasvezes, àsegunda etapa da indexação. No caso em questão, o foco é o ensino da análise de assunto considerada comoa primeira etapa.

Paratanto,fez-seumabuscaexploratórianasbases dedadoseletrônicasnoPortaldePeriódicos da Coordenação de Aperfeiçoamento de Pessoal de Nível Superior - CAPES, a saber: (1) Library Information Science Abstratcs - LISA; (2) SocINDEX; (3) Base de Dados em Ciência da Informação BRAPCl; (4) Scopus e Web Of Science, utilizando-se as seguintes expressões de busca: ("Ensino de Análise de assunto" OR " Teaching Subject analysis" OR “ Lo enseñanza al Análisis documental" OR “Ensino da indexação" OR “Teaching indexing” OR La enseñanza de la Indización). 
Para seleção dos 58 textos recuperados, foram utilizados três critérios: (1) documentos que tratavam do ensino da Análise de assunto; (2) documentos que tratavam do ensino do processo de indexação como um todo, e que poderiam terinformações em potencial sobre a primeira etapa; (3) documentos nos quais os termos "ensino" ou "formação" agregados aos termos "análise deassunto"e"indexador"estivessem notítuloou naspalavras-chaves.Otrabalho resultouem 12 artigos que tratam, especificamente, sobreo ensino da indexação/análise de assunto, sendo cinco na língua inglesa e sete artigos em português.

Observa-sequeumadasquestõesdeescritaquantoaosresultadosdasreflexõessobreosestudos realizadosnoâmbitodeum projeto depesquisaéanecessidadedesempreter-sequecontextualizaro tema,recorrendoàmesmabibliografiadoembasamentoteóricometodológicodoprojeto,oqueleva, muitas vezes, àutilização das mesmasinformaçõesjá referenciadas em outras publicações anteriores. Assim, neste artigo, que tem um cunho mais educacional, antes de contextualizar o processo e o ensino de análise de assunto, apresenta-se uma breve discussão sobre "o que é assunto?", ancorada na literatura da área.

\section{ANÁLISE DE ASSUNTO}

\subsection{0 que é assunto?}

No dicionário de Biblioteconomia e Arquivologia de Cunha e Cavalcante (2008, p. 35), os autoresdefinemassuntocomo"1.Qualquerconceitoou combinaçãodeconceitosconsideradoscomo um todo, conhecido como tema e tópico; 2 . Matéria sobre a qual se fala ou se escreve; 3 . Matéria de que trata um documento."Em geral, o assunto representa o conhecimento humano por meio de um tópicooutemadeumtrabalhodeclaradoexplicitamentenotextooutítuloemquestãoouimplícitoem sua mensagem.

Tanto na literatura estrangeira quanto na literatura nacional, é percebida uma variação terminológicadacompreensãosobreoconceitodeassunto,pornãoserumadefiniçãofácildeentender. Metcalfe (1973) afirma que o uso ambíguo do termo assunto na área de Biblioteconomia e Ciência da Informação causa "conflitos e confusões de significado, particularmente com distinções de geral e específico, e de objeto e aspecto" (METCALFE, 1973, p. 336); concordam com ele também os autores Todd (1992) e Giasson (1993). Por isso, o conceito de assunto ganhou várias conotações diferentes 
na literatura, como: conteúdo, tema, tópico e aboutness; e tem sido um objeto nuclear de estudo na Biblioteconomia e Ciência da Informação em diferentes contextos.

Um dos primeirosautoresa sepreocuparem estudaro significado etrazer uma sistematização para formação de assunto foi Cutter (1904). No Quadro 1, apresentam-se algumas definições, selecionadas na literatura, para elucidar o conceito de "assunto".

Quadro 1 - Definições sobre o conceito "assunto" (continua)

\begin{tabular}{|c|c|}
\hline DEFINIÇÕES & AUTORES \\
\hline $\begin{array}{l}\text { Tema ou tópico do recurso, declarado em seu título ou não, que } \\
\text { recebe um nome que, por sua vez, representa um consenso distinto } \\
\text { de uso. }\end{array}$ & Cutter (1904) \\
\hline $\begin{array}{l}\text { São coisas em geral, reais ou imaginárias, e as condições associadas } \\
\text { a elas. "Os assuntos de nossa observação e raciocínio são coisas em } \\
\text { geral, reais ou imaginárias, e as condições a eles associadas. Vamos } \\
\text { chamá-los de concretos e processos, respectivamente [...]." }\end{array}$ & Kaiser (1911) \\
\hline $\begin{array}{l}\text { É o tema ou tópicos sobre o qual (os quais) os livros, partes de livros, } \\
\text { artigos ou partes de artigos são escritos; um agregado complexo de } \\
\text { aspectos específicos; um composto de termos elementares. }\end{array}$ & Vickery (1953) \\
\hline $\begin{array}{l}\text { É um simples conceito ou tema isolado "que pode ser perfeitamente } \\
\text { guardado em uma única gaveta no vasto gabinete do conhecimento" } \\
\text { (p. 139). }\end{array}$ & $\begin{array}{l}\text { Clas s if ic ation } \\
\text { Research Group } \\
(1957)\end{array}$ \\
\hline $\begin{array}{l}\text { É o corpus de conhecimento organizado que evoluiu no decorrer das } \\
\text { mudanças sociais. }\end{array}$ & Drake (1960) \\
\hline
\end{tabular}

Fonte: Elaborado pela autora, 2020. 
Quadro 1 - Definições sobre o conceito "assunto" (conclusão)

\begin{tabular}{|c|c|}
\hline DEFINIÇÕES & AUTORES \\
\hline $\begin{array}{l}\text { Um corpo de ideias organizadas ou sistematizadas, cuja extensão } \\
\text { e intensão devem ser coerentes com o domínio de interesse e } \\
\text { confortavelmente ajustadas à competência intelectual e ao campo } \\
\text { especializado de uma pessoa qualquer. }\end{array}$ & Ranganathan (1967) \\
\hline $\begin{array}{l}\text { É algo atribuído a documentos ou a outros objetos, mas não algo com } \\
\text { existência independente para além dessa atividade atribuível. }\end{array}$ & Metcalfe (1973) \\
\hline $\begin{array}{l}\text { É um corpo organizado ou sistemático de ideias. Pode ser uma ideia ou } \\
\text { uma combinação de várias... }\end{array}$ & Gopinath (1976) \\
\hline $\begin{array}{l}\text { São os focos de uma publicação, os temas centrais para os quais a } \\
\text { atenção e os esforços do autor foram direcionados. Eles são os aspectos } \\
\text { de uma obra que contêm novas ideias, explicações ou interpretações. E } \\
\text { todos devem ser indexados. Ea literatura mais recente parece usar o termo } \\
\text { aboutness como sinônimo do termo assunto de um documento. }\end{array}$ & Borko e Bernier (1978) \\
\hline $\begin{array}{l}\text { [Um assunto] referia-se [...] àquelas intelecções [...] que haviam recebido } \\
\text { um nome que representava um consenso distinto no uso "e: a" estrutura } \\
\text { sistemática de assuntos estabelecidos "é" residente na esfera pública" }\end{array}$ & Miksa (1983) \\
\hline $\begin{array}{l}\text { É a abstração da ideia geral incorporada no conteúdo do assunto de uma } \\
\text { determinada unidade literária. }\end{array}$ & Coates (1985) \\
\hline $\begin{array}{l}\text { Qualquerconceitooucombinaçãodeconceitosquerepresentamumtema } \\
\text { em um documento. }\end{array}$ & ISO (1985) \\
\hline $\begin{array}{l}\text { Afirma que o termo assunto foi usado exclusivamente em dois sentidos } \\
\text { distintos invariavelmente. Esses dois significados distintos estão relacionados } \\
\text { às duas perguntas realizadas em relação a uma obra: O que é? e sobre o que } \\
\text { é?, ou seja, a forma do conhecimento e o tópico do conhecimento. }\end{array}$ & Langridge (1989) \\
\hline $\begin{array}{l}\text { Éa concepçãoingênua;idealismo subjetivo; idealismoobjetivo; oconceito } \\
\text { pragmático de assunto; e uma teoria do sujeito realista/materialista; são } \\
\text { seus potenciais informativos. }\end{array}$ & Hjørland (1992) \\
\hline $\begin{array}{l}\text { É uma relação entre cada indivíduo e os rabiscos que constituem o } \\
\text { documento. }\end{array}$ & O'Connor (1996) \\
\hline $\begin{array}{l}\text { São termos relacionados ao método e gênero envolvidos no tópico e esses } \\
\text { termos são considerados como a descrição do assunto de um documento. }\end{array}$ & Stam (2000) \\
\hline $\begin{array}{l}\text { 1. Qualquer conceito ou grupo de conceitos tratados explícita ou } \\
\text { implicitamente na mensagem de um documento. } 2 \text {. A síntese dos tópicos } \\
\text { de um documento, expressa por título de assunto. }\end{array}$ & Wellish (2000) \\
\hline $\begin{array}{l}\text { É aquele "algo" que a análise e a recuperação do assunto supostamente } \\
\text { identificam. Isso está intimamente relacionado às perguntas para as quais } \\
\text { um documento deve fornecer respostas. }\end{array}$ & Hjørland (2001) \\
\hline $\begin{array}{l}\text { Um campo de interesse ou atividade; também, o conteúdo de um } \\
\text { documento individual. }\end{array}$ & Broughton (2004) \\
\hline $\begin{array}{l}\text { É um segmento do universo do conhecimento e possui todas as } \\
\text { características que um segmento possui. }\end{array}$ & Sen (2009) \\
\hline Éconcebidocomouma entidade conceitual embutida em um documento. & Dutta (2015) \\
\hline
\end{tabular}


Em suma, Dutta $(2015$, p. 255) apresenta três componentes fundamentais na formação de assunto: (1) conteúdo: assunto real tratado nele; (2) contexto: o ambiente no qual o assunto está inserido; (3) conceito: o tema principal que representa o assunto.

Ranganathan (1967, p. 82) esclarece que todo assunto é oriundo de um assunto básico e seu componente vem de um ou mais conceitos isolados, formando assim oassunto composto. O assunto básicoé um assunto sem nenhuma ideia isolada como componente. Aideia isoladaéalgumaideia ou complexo de ideias, moldada para formarumcomponentedeassunto;mas, semseu contexto, elanão é considerada um assunto.

A partir dessas definições, nota-se a complexidade existente na compreensão da definição de assunto, mas todas levam à concepção de significado, interpretação e ideia. O assunto representa o resultadofinaldeumexperimento,observaçãoepensamentoparaformarqualquerentidadelógica,em alguma formabemdefinidana mentehumana, revelando-sedeváriasmaneiras, formaseestilos-que podem ser uma teoria, algum processo, fenômeno ou aplicação. A seguir, apresenta-seo processo de análise de assunto, a primeira etapa da Indexação.

\subsection{Análise de assunto}

Existe na literatura da área da Biblioteconomia e Ciência da Informação uma variação terminológica em relação ao termo análise deassunto, quetambém pode ser denominado deanálise temática ou análise conceitual; entretanto, nesta pesquisa, o termo análise de assunto éo que melhor representa o processo de representação de conteúdo de um documento.

Análise de assunto, a primeira etapa da indexação, é considerada por Foskett (1973, p. 40) como "a operação chave da indexação, que é a decisão sobre o que o documento é, e ainda é menos discutida e a menos reduzível a regras". Cesariano e Pinto (1980) destacam que a análise de assunto é a operação-base para todo o procedimento de recuperação de informação. Uma definição muito próxima da definição dessas autoras é dada por Naves (2000, p. 249), quando descreve a análise de assunto como uma operação base da indexação de assuntos, compreendendo o processo pelo qual passa o indexador para extrair o conteúdo de um documento.

De acordo com Hutchins (1978, p. 172), "um problema crucial na área da Ciência da Informação diz respeito à identificação do assunto de um documento". Concordando com este autor, Wellisch (1992, p. 69) ressalta que o processo de"indexação pode ser retratado como uma 
arte", pelo seu grau de complexidade, sendo considerado por Weinberg (2017, p. 1978) "uma arte e não uma ciência".

De acordo com os Princípios do Unisist (1981, p. 8), a etapa análise de assunto possui três estágios:(1)compreensão doconteúdo dodocumento comoumtodo;(2)identificaçãodosconceitos que representam esse conteúdo e (3) seleção dos conceitos válidos para recuperação, sendo que se destaca que, "na prática, esses três estágios se superpõem".

Ofinal do estágioéindicado com a definição da chamadafrase deindexação, sendoelaborada peloindexadoremLinguagem Natural(LN).Apóstodooprocessointelectualdeleituraecompreensão dotexto, deidentificação eseleção de conceitos representativos do documento em foco, oindexador deve afirmar: "Este documento trata de..." A partir dessa definição, o indexador pode passar para a etapa final do processo de indexação, a tradução da análise de assunto em termos de indexação.

Análise de assunto é uma atividade intelectual, portanto, subjetiva, que se inicia pela leitura técnicadasprincipaispartesdeum documento, aqual exigedoindexadorconhecimentoslinguísticos, cognitivose lógicos para serrealizada, para determinardeque trata um documento, istoé, qualéoseu assunto. Porisso, torna-se um processo desafiador para os indexadores, exigindo desses profissionais procedimentos subjetivos por se tratar de uma atividade intelectual. Para tanto, fazer a leitura para a compreensão e a identificação do texto é primordial, para, posteriormente, realizar a seleção dos termos válidos para a representação do conteúdo de um documento.

O grande dificultador na realização da atividade da análise de assunto é que esta atividade envolve processos cognitivos complexos para a interpretação do conteúdo de um documento, e, na seleção dos termos que melhor o representa, os aspectos linguísticos relacionados à coesão, à coerência e à estrutura textual, e os aspectos lógicos que ocorrem na interpretação e na inferência de tomada de decisão são totalmente subjetivos, e dependem de vários fatores, como a experiência do indexador, o ambiente de trabalho, as normas institucionais às quais o indexador está sujeito, os instrumentosutilizados,oconhecimentosobreodomíniododocumento,entreoutros.Bernier(1965) afirma que“identificação de assunto é difícil de ensinar e aplicar, especialmente quando os assuntos são complexos ou implícitos. A pessoa sem conhecimento no campo indexado acha impossível a identificação consciente de assuntos" (BERNIER, p. 324, 1965).

Noentanto, nota-sequeosestudiosos daindexação norteiamseusestudos para aslinguagens controladas, gerando uma ausência naqueles que tratam da primeira etapa do processo. Por isso, a importância do ensino da Análise de assunto nos cursos de Graduação em Biblioteconomia, que 
é necessário, para que sejam formados profissionais com capacidade para fazer essa análise com qualidade e eficiência.

\section{ENSINO DE ANÁLISE DE ASSUNTO}

Em geral, nossos alunos são ensinados a seguir diretrizes na realização do processo de análise de assunto centrada nos atributos dos documentos, sem as considerações sobre as necessidades dos usuários e o contexto no qual a análise é feita, o domínio em que o documento está inserido, identificandoascaracterísticasespecíficas docampodeconhecimento, sejamelas deordem cultural, terminológica, histórica ou linguística.

Desde o final da década de 1960, existe uma preocupação em relação ao ensino da indexação, conforme afirma Wilson (1968, p. 73):"é curiosa a falta de informação para o profissional sobre como identificar o assunto". Geralmente, ensina-se a focar nos atributos do documento em vez de explicar ao indexador q que deveser realizado para a extração do assunto. Outro autor que concorda com essa perspectiva é Mai (1997), que enfatiza que"[... o objetivo é determinar o assunto do documento e não os usos potenciais do documento] ... Isso sugere que é quase impossível formular diretrizes sobre como determinar o assunto de um determinado documento"2 (MAI, 1997, p. 60, tradução nossa). Hjorland (1997) concorda que essas diretrizes são mais focadas nas características do documento, sendo dependentes daanálise do indexador, oquetrazproblemas naanálisedeassunto, poisnãoleva em consideração o contexto e as necessidades dos usuários.

\subsection{Reflexões a partir da literatura}

Percebe-se,deacordocomaliteratura,quenãoexisteumametodologiadetalhadadecomofazer análise de assunto, pela sua subjetividade, e muito menos para seu ensino. Nesta seção, apresentase como o ensino da análise de assunto/indexação tem sido abordado a partir da literatura específica sobre o tema, e traz-se a experiência da autora a partir das reflexões no âmbito da sua pesquisa e ensino da disciplina.

O primeiro artigo recuperado na literatura foi o de Kashyap (1975), no qual o autor não aborda especificamente o ensino do processo da análise de assunto, mas trata da necessidade de ensinar

2 "[...] theaim isto determinethesubject of the documentand not the potencial uses of the document]...Thissuggest that it is almost impossible to formulate guidelines on how to determine the subject of a given document. " 
aos alunos a compreensão do que é um conceito, ao invés de memorizar, para que os alunos possam desenvolver a habilidade de raciocinar ejulgar, sendo esta uma das principais qualidades requeridas do profissional indexador. Como exemplo, o autor reflete sobre o ensino da teoria da classificação na biblioteconomia.

Em 1984, Götz Greiner, em seu artigo "Some reflections on teaching subject analysis in the field of documentation" (GREINER, 1984), aponta que a análise de assunto é um dos processos mais importantes em um SRI, necessitando, por isso, ser uma atividade mais bem compreendida, e ao mesmotempoterum treinamentomais detalhado, paragarantirumensinoeficiente.Paraisso,oautor afirma a necessidade de haver um curso bem estruturado, composto de temáticas que possibilitem o aprendizado, e o desenvolvimento de habilidades necessárias para realizar a atividade de análise de assunto. Como método, o autor sugere (1) discussão da bibliografia da disciplina, (2) exercícios individuais ou em grupos, ou a combinação de ambos, (3) construção, uso e avaliação de sistemas de classificação e tesauros, (4) seminários sobre tópicos específicos, e, ao final, (5) a realização de um projeto, como por exemplo, a construção de um tesauro ou uma base de dados de resumos de jornais para praticar a abstração e a indexação.

Nohr (1991), refere-seà necessidade do ensino/treinamento do bibliotecário na formação em análise de assunto tendo como influências diversas áreas do conhecimento, como, por exemplo, as inovações tecnológicas. Nesse caso, as contribuições advindas desta perspectiva devem possibilitar uma instrução voltada para ofuturo, além defornecer uma base teórica sólida, especialmenteàluzdosdesenvolvimentostecnológicos, eparatreinamentodosalunos notocante à tomada de decisão. Assim, o autor considera que o ensino da análise de assunto deve se pautar em duas influências específicas: (1) a orientação teórica baseada nos estudos sobre os sistemas de conceitos, a teoria da classificação, linguística e filosofia, entre outras áreas; e (2) a orientação prática, cuja prática da análise deassunto, porserna década de 1990, baseava-senouso doCódigo de Catalogação Anglo Americano como instrumento central e cooperativo e no crescimento das aplicações tecnológicas, como o caso do aumento da disponibilização dos catálogos de acesso público on-line (Online Public Acess Catalog - OPACs). Norh (1991, p. 153) conclui que 
Detalhando um pouco mais sobre o currículo do curso de biblioteconomia, Nohr (1991) sugere que as disciplinas sejam divididas em básicas e avançadas, e que alguns fundamentos teóricos devam ser ensinados no início do curso, para formar a base de conhecimento para o que será ensinado aos alunos posteriormente. Assim, o que é difícil, nesse caso, é especificar qual parte teórica será inserida no conteúdo da disciplina Análise de assunto, visto que teoria e prática não podem serensinadas separadamente, e são utilizadas em todas as atividades de análise de assunto, ao longo do curso.

Anderson (2002) parte do pressuposto de que o processo de indexação, principalmente a análise de assunto, não pode ser ensinado, por ser um procedimento que exige raciocínios lógicos e cognitivos, porém, geralmente é direcionado por meio de normas eguias que orientam o passoa passo para a realização do processo. O autor ressalta que a indexação não pode ser reduzida a um conjunto de etapas a serem seguidas; assim, procura, no início do ensino da disciplina, fornecer aos alunos um panorama geral do conteúd o sobre indexação, para auxiliá-los na compreensão de como tomar a decisão para fazer as melhores escolhas dos termos que representam o conteúdo do documento.

Somado a isso, o autor sugere uma lista de 20 tópicos considerados por ele essenciais, levantados aolongo dosanos de sua experiência de indexadore professor, os quaisoauxilia noensino de indexação. Ele os utiliza como se fossem etapas a serem seguidas, para que, ao final da disciplina, 0 alunopossaterumamelhorcompreensãodotodo. Issosematerializaemum projetonoqualosalunos fazem uma análise dessas temáticas no contexto de um Sistema de Recuperação da Informação(SRI), e apresentam os resultados ao final da disciplina. Cada tópico é descrito detalhadamente, conforme apresentados sequencialmente pelo autor:

(1) escopo do assunto e o contexto do domínio,

(2) tipologia documental,

(3) domínio do documento,

(4) interface de busca,

(5) unidades documentais,

(6) matéria indexável,

(7) métodos de análise de assunto,

(8) exaustividade da indexação,

(9) especificidade dos termos de indexação, 
(10) índices manuais, eletrônicos e para navegação,

(11) sintaxe de termos para representação e recuperação de informação (string indexing),

(12) controle e gerenciamento de vocabulário,

(13) substituição de mensagens e textos,

(14) localizadores e links entre substitutos e documentos,

(15) aparelhos alternativos para acesso à informação (surrogate displays),

(16) organização de índices em ordem alfabética, relacional, híbrido, disponibilizados em forma impressa e navegáveis,

(17) elaboração de impressos de índices de final de livro,

(18) interfaces de pesquisa e visualização de informação,

(19) estruturação e organização dos registros de metadados,

(20) diferentes tipos de displays para diferentes propostas, como, por exemplo, acesso ao texto completo.

Outro artigo, também publicado em 2002, foi o de Sylvia Coates (COATES, 2002), no qual ela aborda as necessidades de desenvolver habilidades cognitivas para realizar o processo de indexação e seleção de termos de forma eficiente. A autora questiona se esse processo pode ser ensinado, quais habilidadessãonecessárias, oqueestáenvolvidoemseu ensinoequala responsabilidadedoaluno na aprendizagem. Aponta que, para isso, éimportante que o professor, ao ensinar esse conteúdo, tenha competência experiência, vistoquenem todososindexadores sãobonsprofessores. Arealizaçãode umaboaanáliseeseleçãodetermosdependemuitodacompreensãodeleitura, relacionadaàsquestões cognitivas, estruturastextualesemântica.Dosalunos, éesperadoquetenhamuma boacompreensão deleituraeashabilidades deconceituação para determinarerelacionarosconceitos, sendoesses dois processos essenciais para serem bons indexadores e para os professores serem capazes de articular o ensino do processo de forma interativa. Os aspectos que são considerados importantes no ensino, mais especificamente, são:

(1) ensino do processo convencional de indexação,

(2) elaboração de índices considerando a especificidade dos termos,

(3) bibliografia atualizada sobre a temática, e

(4) exercícios para auxiliar os estudantes a entenderem a prática da análise e seleção de termos, que devem ser corrigidos pelos professores e retornados aos alunos com as correções. 
Com isso, o processo torna-se colaborativo tanto da parte do professor quanto da parte do aluno, eissocontribuipara queosalunosaprendamas diversas abordagensno processo deindexação e no refinamento do processo.

Fourie (2002) observa que os alunos de graduação geralmente não têm ainda experiências nem conhecimento substancial e, com isso, ainda possuem um vocabulário limitado. Como os procedimentosdeindexaçãoeresumorequeremconhecimentoalémdoencontradonoconteúdode um documento, a autora propõe um modelo para o ensino de indexação e elaboração de resumos com base na abordagem sociocognitiva com oito atividades planejadas para orientar os alunos nas atividades da disciplina, em forma de portfólio. Para sua realização, propõe-se criar um ambiente em que se deve (1) dividir os alunos em pequenos grupos, (2) atribuir a cada grupo a responsabilidade de realizara representação da informação em umaárea doconhecimentoe deum tipodeSRI,(3) garantir queasrepresentações sejam adaptadas àsnecessidades, habilidadescognitivas eaovocabulário dos alunos, (4) indicar qual metodologia foi utilizada para coletar os termos e o resultado, (5) realizar uma análise comparativa entre os resultados de todos os grupos, para recomendar melhorias nos tipos de SRI. Assim, os alunos podem realizar o processo de indexação e resumo do ponto de vista teóricoprático, coma abordagem sociocognitiva na representação da informação noâmbito da pesquisa em um SRI; permitindo, nesse caso, que os alunos adquiram experiências na indexação não somente na concepção orientada ao conteúdo e ao sistema, mas também na concepção orientadaà demanda do usuário. Porém, Fourie (2002, p. 85) acrescenta que “... as sugestões mencionadas acima são muito básicasepodemservistascomoum pequenopassoem direçãoaumaabordagemsociocognitivapara ensino de indexação e resumo em nível de graduação" (FOURIE, 2002, p. 85).

Nesse contexto, os portfólios - considerados como conjunto de trabalhos de pesquisa em que o aluno demonstra seus esforços e desenvolvimento na seleção de conteúdo, nos critérios de seleção, sendo mais participativo do que avaliado, evidenciando sua autonomia e motivação para tornar-se independente no processo de aprendizagem - têm sido usados com sucesso no ensino das habilidades de pesquisa de informação em diversas disciplinas. Eles são compostos geralmente de diversas tipologias documentais, tanto daárea específica queestá sendoestudada no projeto quanto na área de documentos que possam subsidiar o estudo sobre indexação e resumos (FOURIE; VAN NIEKERK, 2001).

Sabe-se que é difícil o aluno vivenciar em sala de aula todas as atividades previstas em um SRI, para que possa transportar essa experiência para a prática da indexação. Segundo Fujita (2004), 
ocontextodoprofessornoensinodeleituradocumentária paraindexaçãoénorteado pordificuldades inerentes à própria escassez de metodologias de indexação. A partir dessa constatação da autora, sua pesquisa passa a ser direcionada para investigação de estratégias de ensino, que culmina em umapropostadeaplicaçãodeum ModelodeLeituraDocumentáriaparaaprimoramentodoensinode indexação.

Fujita e Rubi (2006) publicam um modelo de leitura documentária para a indexação de artigos científicos que visou à formação de indexadores das bibliotecas das Universidades Estaduais Paulistas - USP, UNESP e UNICAMP, em curso de Educação a Distância. As autoras justificam o desenvolvimentodessemodelo, baseadonousodeestratégias deleitura, peladificuldadedoindexador frente à complexidade da realização do processo de análise de assunto. Segundo Fujita e Rubi (2006, p. 2):

Nesse sentido, tanto os cursos degraduaçãoem Biblioteconomiaquanto oscursosquevisam àeducaçãocontinuadadoprofissionalindexadortêmumagrandeparceladeresponsabilidade na formação e capacitação do indexador que necessita dos aportes teórico-metodológicos específicos sobre leitura documentária, dotados dos aspectos cognitivos e linguísticos (FUJITA; RUBI, 2006, p. 2).

OModelofoielaboradoconsiderando-seaestruturatextual paraaidentificaçãodeconceitospor meiodequestionamentoscombinandoassistemáticas deidentificaçãodeconceitosanáliseconceitual, na primeira coluna, eabordagem sistemática da ABNT (1992), na segunda coluna, e coma localização dos conceitosem parte da estrutura textual, na terceira coluna. As autoras concluem que o Modelo de Leitura pode ser considerado como uma proposta que auxiliará o processo de indexaçãoe, também, a formação e a capacitação do indexador no desempenho da análise de assunto. Porém, Fujita e Rubi (2006) ressaltam que:

... o uso do Modelo de Leitura deve ser, necessariamente, acompanhado dos fundamentos teóricos e metodológicos deanálise de conteúdos documentários e deleitura documentária para que a concepção de análise de assunto do Modelo de Leitura tenha sentido em um contexto de tratamento documentário (FUJITA; RUBI, 2006, p. 12).

ParaFujita(2007),éimportante, noprocessodeensinodeleituradocumental,noâmbitodasala deaula, desenvolverestratégias deensinoa partir da abordagem cognitiva no contexto pedagógico, que estimulem o desenvolvimento de conhecimentos profissionais prévios dotados de estratégias de leitura documentária para contextos de sistemas de recuperação de informação. Para a autora, o processo de análise é iniciado pela leitura do texto, mas mais especificamente por estratégias de leitura aplicadas, porém sem procedimentos específicosque atendam àtipologia textual.Com isso, a 
qualidade do processo é influenciada; o uso do modelo minimiza essa questão, possibilitando que o aluno, aoutilizá-loemsaladeaula,tenhacondiçõesdesimularotrabalhoprofissionalmaisadequadoà realidade dos SRIs. Para auxiliar nesse processo, Fujita (2007, p. 408) sugere três orientações a partir daabordagemcognitiva,eduasorientaçõesapartirdaabordagemsociocognitivaafimdefundamentar as estratégias de ensino de leitura documentária.

Em 2008, Fujita e Ferreira apresentam, no IBERSID, o produto de um trabalho no qual relatam o resultado da aplicação do modelo de leitura documentária no ensino do processo deanálise de assunto para indexação. A pesquisa foi realizada com os alunos dos Cursos de Biblioteconomia da Universidade Estadual Paulista-UNESP-Campus de Marília e da Universidade Federal do Rio Grande doSul-(UFRGS, noBrasil.Apartirdosresultados, foigeradaumaavaliaçãocomparadaquepropiciou reflexõesconjuntas entre professores e alunos sobre o processo de análise de assunto. Ficou evidente a importância do ensino dos aportes teóricos metodológicos oriundos da cognição e da linguística para a formação do bibliotecárioindexador,principalmenteparaodesempenhodaleituradocumentária.Alémdisso,Fujitae Ferreira (2008) sugerem que os professores queensinam indexação na graduação em Biblioteconomia adotem o Modelo de Leitura Documentária, para que ele seja avaliado e divulgado com o intuito de demonstrar a importância da leitura documentária no processo da representação da informação.

Fujita (2009) realiza outra pesquisa sobre o ensino do indexador nos estágios iniciais do aprendizado,comumaaplicaçãopedagógicadomodelodeleituradocumentáriaapartirdaabordagem sociocognitiva. Utilizou-se da técnica de coleta de dados do protocolo verbal interativo com pares de alunos com o intuito de auxiliá-los na compreensão do processo de indexação. A autora descreve a importância de considerar o modelo interacionista de Giasson (1993), no qual o autor considera três variáveis que se interagem no momento da leitura: o leitor, o texto, e o contexto. Para Fujita (2009, p.432), “o contexto, particularmente, tem importância preponderante durante a leitura porque fixa o objetivoedelimitaodesempenhodoindexador".Issoauxiliaoalunoaanteverocontextoprofissionale estratégiasadequadasaoprocessodeanálisedeassunto,vistoqueoobjetivodaleituradocumentáriaé diferentedoobjetivodaleituracomum, poiselatemcomofinalidadeatenderaosobjetivosdaindexação. Ao final, a autora, mais uma vez, constata a necessidade de os currículos voltados para a formação de indexadoresincluíremconteúdosrelativosàleituradocumentária,commetodologiasdeaprendizagem de abordagem sociocognitiva no processo de leitura com o uso da técnica dos protocolos verbais.

Seguindo essa pesquisa, Fujita (2010) relata os resultados da aplicação das propostas do PortfóliodeFourie(2002)eoprincípio dacontextualizaçãosocialdeCiênciadalnformaçãodeHjørland 
(2002) junto à disciplina "Indexação" do Curso de Biblioteconomia da UNESP de Marília. A autora, tambémnessapesquisa, utilizadatécnica decoletadedadosdoprotocoloverbalinterativo,aplicando: (1)a dinâmica em grupo fora de sala, para elaboração do portfólio com informações sobre a política de indexaçãoerecuperaçãoBibliotecadaUNESP,esobreademandadasnecessidadesdospesquisadores daUNESP;(2) a dinâmica dentroda sala deaula com pares dealunos para indexarartigos de periódicos científicos, e livros. Após a realização dessas duas dinâmicas, surgiram as dificuldades dos grupos de alunos em sala de aula quanto a:

...nãofamiliaridadecomoassunto dotextoetermosespecíficos, ausência deconhecimentos teórico-práticosdoprocessodeindexação,falta doobjetivodeindexação, desconhecimento do contexto de busca de um sistema de informação com demandas reais de informação pela comunidadedeusuáriosefaltadeuniformidadedeprocedimentosdeanálisedeassuntopara identificação de termos representativos (FUJITA, 2010, p. 92).

Para solucionar essas dificuldades, a autora sugere que os alunos conheçam, primeiramente, as necessidadesdosusuáriosnocontextodoSistemadeRecuperaçãodalnformação(SRI),para,posteriormente, aprendermetodologiasdeanálisedeassuntoparaindexação.Aofinal,conclui-sequeametodologiapropostafoi válida, e que as duas dinâmicas deram aos alunos autonomia na tarefa de indexação.

Em 2017, Dal'Evedone e Fujita realizam a pesquisa "O ensino da indexação no Brasil" com o intuito de conhecer os conteúdos e as práticas pedagógicas utilizadas pelos docentes no ensino da indexação dos cursos de Biblioteconomia existentes nas Universidades Federaise Estaduais, naquela época, no Brasil. Para isso, foram coletadas e analisadas informações sobre os currículos e a grade curricular, concluindo que os planos de ensino proporcionam aos alunos:

... conhecimentos teóricos e metodológicos concernentes aos princípios e ao processo que Ihespermitamcompreender, aplicarecontextualizararepresentaçãodeassuntoemsistemas de recuperação da informação, sendo requisito dar a conhecer a prática de indexação e elaboração de índices (DAL'EVEDONE; FUJITA, 2017, p. 6).

No artigo"Formação do bibliotecário indexador no Brasil: análise dos aspectos temáticos em planos de ensino", Dal'Evedone e Fujita (2017) publicam os resultados dessa pesquisa, concluindo quenão existeuma metodologia padronizada;porém ressaltamqueasmetodologiasquecombinam a teoria e a prática, como os exercícios realizados em sala de aula, auxiliam os alunos a assimilarem melhoroconteúdo programáticoda disciplina. Ressaltamaindaqueháanecessidadeeaimportância de manterem-se pesquisas sobre as práticas pedagógicas da indexação, considerandoessa temática essencial para a formação do profissional bibliotecário; sendo, portanto, essencial inseri-la nos currículos do curso de Biblioteconomia. 


\section{CONSIDERAÇÕES FINAIS}

Esteartigotrouxe resultados parciais dereflexões realizadas noâmbitodo projetode pesquisa PQ-CNPq sobre“Estudo sobre o estatuto teórico metodológico da análise de assunto", que tem como objetivoprincipalestudarosfundamentosteóricosmetodológicosdaanálisedeassunto, verificando, principalmente,a evoluçãometodológicaequaissãoascontribuiçõesdepesquisasquevisammelhorar a subjetividade desse processo e contribuir para o ensino e a aprendizagem da disciplina Análise de assunto na graduação.

A indexação/análise de assunto é um dos processos mais importantes em SRI para garantir a recuperação da informação e o acesso a ela, mas nem sempre o indexador possui uma formação especializadanaáreadetratamentodeconteúdosdedocumentos,tantodopontodevistatécnicoquanto do teórico. Sendo assim, é um processo que deve ser ensinado e aprendido pelos bibliotecários em todos os seus aspectos, por ser uma formação especializada, no âmbito do curso de Biblioteconomia.

Como intuito de elucidar o conceito de"assunto", pela sua complexidade, apresentaram-se 21 definições de autores seminais da área, no período de 1904 a 2017, as quais levam à concepção de significado, interpretaçãoeideia, podendoserreveladosdediferentesmaneiras,formaseestilo, sendo resultado de um processo, fenômeno ou aplicação.

A partir da análise realizada na literatura específica sobre o ensino da análise de assunto/ indexação, conclui-seque nãosãomuitososestudos realizados, principalmentecomfoco na primeira etapa, análise de assunto; tanto que oresultado da pesquisa bibliográfica nãofoi expressivo. Aotodo, seisautoresinternacionaise, noBrasil,majoritariamenteartigosdeautoriaecoautoriada/comaprofa. MariângelaFujita, resultadosdeseuprojetodepesquisacomoPesquisadoradoCNPq,edeorientações na pós-graduação, a partir de estudos sobre leitura documentária. Desses estudos, a maioria dos autores não apontam uma metodologia detalhada para o ensino da análise de assunto. Ressalta-se o trabalhodeFourie(2002), noqualaautorapropõeum modeloparaoensinodeindexaçãoeelaboração de resumos com base na abordagem sociocognitiva com oito atividades planejadas para orientar os alunos nas atividades da disciplina, em forma de portfólio; e o Modelo de Leitura Documentária proposto por Fujita e Rubi (2006).

Porém,em relaçãoaoensinodaAnálisedeassunto, perdurammuitaslacunasaserem resolvidas, dentre elas: (1) como minimizar esta carga subjetiva no ensino e aprendizagem do processo; (2) como ensinar o indexador a realizar este processo seguindo as diretrizes, sem perder de vista o contexto do 
documento e as necessidades dos usuários; (3) como ensinar os alunos do curso de Biblioteconomia a aprenderesseprocesso, quedemandaumacargacognitivaeumforteentendimentodaestruturatextual.

Isso posto, notou-se umaunanimidadeentresosautores em relaçãoàs dificuldades deensinar a Análise de assunto pelo seu caráter subjetivo, e que, por conseguinte, requer do aluno raciocínios lógicos e cognitivos, além de conhecimento da linguística e tipologia documental.

Pode-se dizer que é possível melhorar o ensino da Análise de assunto, desde que existam responsabilidades do professor no ensino e do aluno na aprendizagem desse processo.

Assoluçõesvislumbradas, resumidamente, perpassam(1)pelocontínuoestudodaliteraturada área,(2) pela análise qualitativa das diretrizese metodologiasjá propostas,(3) peloensino pormeio da abordagemsociocognitiva,(4) pelacompreensãodalinguísticatextuale(5)pelasregrasdeinferências lógicas, (6) pelo fornecimento de uma baseteórica sólida, especialmenteà luzdos desenvolvimentos tecnológicos.

Assim,ficouevidenteanecessidadedeseterumcurso bemestruturado,compostodetemáticas que possibilitam o aprendizado;eo desenvolvimento de habilidades necessárias para a realização da atividade de análise de assunto, tendo um equilíbrio entre a teoria e a prática. Soma-se a isso um trabalho colaborativo tanto da parte do professor no ensino, quanto do aluno na aprendizagem, para que aprendam as diversas abordagens no processo de indexação e no refinamento do processo.

No âmbito do ensino da disciplina de Análise de assunto, ministrada pela autora, no curso de Biblioteconomia na Escola de Ciência da Informação da UFMG, ressalta-se que, entre alguns dos resultadosjáalcançadosna pesquisa, encontra-seumobjetode aprendizagem,emforma de umjogo pedagógico, que foi desenvolvido com o objetivo de ensinar aos alunos o processo de análise de assuntoecategorização. Essapropostametodológicatemporobjetivotrabalharatomada dedecisão e incentivar o potencial criativo dos alunos, para que aprendam de forma lúdica esses processos tão subjetivos. Esse jogo já foi aplicado nos últimos três semestres (2018-2019), obtendo um resultado positivo dos alunos. Pretende-se que esse resultado seja divulgado posteriormente, somando-seaos outros resultados oriundos do projeto de pesquisa PQ-CNPq.

\section{AGRADECIMENTO}

AoapoiodoConselhoNacionaldeDesenvolvimentoCientíficoeTecnológico(CNPq/Brasil)pela concessão da bolsa de Produtividade de Pesquisa (PQ). 


\section{REFERÊNCIAS}

ANDERSON, D. J. Indexing, teaching of. The Indexer, [S.I.], v. 23, n. 1, p. 2-7, Apr. 2002.

ASSOCIAÇÃO BRASILEIRA DE NORMAS TÉCNICAS. ABNT NBR 12676: métodos para análise de documentos: determinação de seus assuntos e seleção de termos de indexação. Rio de Janeiro: ABNT, 1992.

BERNIER, C. L. Indexing process evaluation. American Documentation, [S.I.], v. 16, n. 4, p. 323-328, Oct. 1965.

BORKO, H.; BERNIER, C. L. Indexing Concepts and Methods. New York: Academic, 1978. 256 p.

BROUGHTON, V. Essential classification. London: Facet Publishing, 2004.

CESARINO, M. A. da N.; PINTO, M. C. M. F. Análise de Assunto. Revista de Biblioteconomia de Brasília, [S.I.], v. 8, n. 1, 1980. Disponível em: <http://hdl.handle.net/20.500.11959/brapci/72529>. Acesso em: 02 dez. 2020.

CLASSIFICATION RESEARCH GROUP. The need for a faceted classification as the basis for all methods of Information Retrieval. In: PROCEEDINGS OF THE INTERNATIONAL STUDY CONFERENCE ON CLASSIFICATION FOR INFORMATION RETRIEVAL, 1957, Dorking. Anais [...]. Dorking: ASLIB, May 1957. p. 137-147.

COATES, E. J. Significance and term relationship in compound headings. In: CHAN, L. M; RICHMOND, P.A.; SVENONIOUS, E. (ed.). Theory of subject analysis: a manual. Littleton, Colorado: Libraries Unlimited, 1985. p. 181-195.

COATES, S. Teaching book indexing: cognitive skills and term selection. The Indexer, [S.I.], v. 23, n. 1, p. 15-17, Apr. 2002.

CUNHA, M. B. da; CAVALCANTI, C. R. de O. Dicionário de Biblioteconomia e Arquivologia. Brasília: Briquet de Lemos, 2008. 451 p.

CUTTER, C. A. Rules for a dictionary catalog. Washington DC: Government Printing

Office, 1904.

DAL'EVEDOVE, P. R.; FUJITA, M. S. L. Formação do bibliotecário indexador no Brasil: análise dos aspectos temáticos em planos de ensino. São Carlos. In: ENREDO - ENCONTRO DE REPRESENTAÇÃO DOCUMENTAL, 1., 2017, São Carlos. Anais [...]. São Carlos: Universidade Federal de São Carlos, 2017.

DRAKE, C. L. What is a subject? Australian Library Journal, n. 9, p. 34-41, 1960.

DUTTA, B. Ranganathan's elucidation of 'subject' in the light of 'Infinity ( $\infty)$.' Annals of Library and Information Studies, [S.I.], v. 62, n. 4, p. 255-264, 2015. Disponível em: <http://op.niscair.res.in/ index.php/ALIS/article/view/11415>. Acesso em: 02 dez. 2020. 
FOSKET, A. C. A abordagem temática da informação. Tradução de Agenor de Briquet de Lemos. São Paulo: Polígono, 1973.

FOURIE, I. How can we take a sócio-cognitive approach in teaching indexing and abstracting? The Indexer, London, v. 23, n. 2, p. 83-85, Oct. 2002.

FOURIE, I.; VAN NIEKERK, D. Follow-up on the use of portfólio assessment for a module in research information skills: an analysis of its value. Education for Information, [S.I.], v. 19, n. 2, p. 107-26, 2001.

FUJITA, M. S. L. A leitura documentária na formação inicial do indexador: a abordagem sociocognitiva na investigação de estratégias de ensino. Marília: UNESP, Faculdade de Filosofia e Ciências, 2004. (Projeto de Produtividade em Pesquisa, CNPq).

FUJITA, M. S. L. La enseñanza de la lectura documentaria en el abordaje cognitivo y socio-cognitivo: orientaciones a la formación del indizador. Anales de Documentación, [S.I.], v. 10, p. 1-16, 2007.

FUJITA, M. S. L. Modelo de leitura documentária para indexação de textos científicos como metodologia de ensino sociocognitiva: análise da aplicabilidade com uso de protocolo verbal com vistas à sua adequação. In: BORGES, M. M.; CASADO, E. S. A Ciência da Informação criadora de conhecimento, [S.I.], v. 1, 2009. p. 431-448.

FUJITA, M. S. L. O contexto profissional do indexador no ensino de indexação. Enc. Bibli: R. Eletr. Bibliotecon. Ci. Inf., Florianópolis, v. 15, n. 30, p. 91-104, 2010.

FUJITA, M. S. L.; FERREIRA, G. I. S. Ensino do processo de análise de assunto para indexação com aplicação de um modelo de leitura. Ibersid, [S.I.], p. 163-176, 2008.

FUJITA, M. S. L.; RUBI, M. P. Um modelo de leitura documentária para a indexação de artigos científicos: princípios de elaboração e uso para a formação de indexadores. DataGramaZero - RevCi. Inf., Rio de Janeiro, v. 7, n. 3, jun. 2006. Disponível em: <http://www.dgz.org.br/jun06/Art_04.ht>. Acesso em: 02 dez. 2020.

GIASSON, J. A compreensão na leitura. Lisboa: Asa, 1993. 317 p.

GOPINATH, M. A. Colon Classification, l: classification in the 1970's. 2. ed. MALTBY, Arthur (ed.). London: Clive Bingly, 1976. p. 51-80.

GREINER, G. Some reflections on teaching subject analysis in the field of documentation. International Classification, [S.I.], v. 11, n. 2, p. 66-68, 1984.

HJØRLAND, B. Epistemology and the sócio-cognitive perspective in information science. Journal of the American Society for Information Science and Technology, [S.I.], v. 53, n. 4, p. 257-70, 2002.

HJØRLAND, B. Information seeking and subject representation: an activity-theoretical approach to information science. Westport: Greenwood Press, 1997. 213 p.

HJØRLAND, B. The concept of subject in information science. J. Doc., London, v. 48, n. 2, p. $172-$ 200, 1992. 
HJØRLAND, B. Towards a theory of aboutness, subject, topically, theme, domain, field, content and relevance. Journal of the American Society for Information Science and Technology, [S.I.], v. 52, n. 9, p. 774-778, 2001.

HUTCHINS, W. K. The concept of "aboutness" in subject indexing. Aslib Proceedings, [S.I.], v. 30, n. 5, p. 172-181, May. 1978.

INTERNATIONAL ORGANIZATION FOR STANDARDIZATION. ISO 5963: documentation: methods for examining documents, determining their subjects, and selecting indexing terms. United States: ISO, 1985.

KAISER, J.O. Systematic indexing. London: Pitman, 1911.

KASHYAP, M. M. Concepts comprehension building in students and teaching of theory of library classification. Internacional Classification, [S.I.], v. 2, n. 1, 1975.

LANCASTER, F. W. Indexação e resumos: teoria e prática. Brasília: Briquet de Lemos, 2004. 452 p.

LANGRIDGE, D. Subject analysis: principles and practice. London: Bowker-Sour, 1989.

MAI, J-E. The concept of subject: on problems in indexing. [S.I.]. In: PROCEEDINGS OF THE 6TH INTERNATIONAL STUDY CONFERENCE ON CLASSIFICATION RESEARCH, 6., 1997, [S.I.]. Anais [...]. [S.I.: S.n], 1997. p. 60-67.

METCALFE, J. When is a subject not a subject? In: RAWSKI, C.H. (ed.). Towards a theory of Librarianship. New York: Scarecrow, 1973.

MIKSA, F.The subject in the dictionary catalog from Cutter to the present. Chicago: American Library Association, 1983.

NAVES, M. M. L. Curso de indexação: princípios e técnicas de indexação, com vistas à recuperação da informação. Belo Horizonte: UFMG, Biblioteca Universitária, 2004. 23 p. Material didático.

NAVES, M. M. L. Fatores interferentes no processo de Análise de assunto: estudo de caso de indexadores. 2000. 273 f. Tese (Doutorado em Ciência da Informação) - Escola de Ciência da Informação, Universidade Federal de Minas Gerais, Belo Horizonte, 2000.

NOHR, Hollger. Training of librarian in Content Analysis. Int. Classif., [S.I.], v. 18, n. 3, 1991.

O'CONNOR, B. C. Explorations in indexing and abstracting: pointing, virtue, and power. Englewood, CO: Libraries Unlimited, 1996. 182 p.

RANGANATHAN, S.R. Prolegomena to library classification. London, Asia Pub. House, 1967.

SEN, B.K. Universe of knowledge from a new angle. Annals of Library and Information Studies, [S.I.], v. 56, n. 1, p. 7-12, 2009.

STAM, R. Film theory: an introduction. Blackwell: Oxford, 2000. 
TODD, R. J. Academic indexing: what's it all about? The Indexer, London, v. 18, n. 2.p. 101-104, 1992.

UNISIST. Princípios de indexação. Revista da Escola de Biblioteconomia da UFMG, Belo Horizonte, v. 10, n. 1, p. 83-94, mar. 1981.

VICKERY, B.C. Systematic Subject Indexing. Journal of Documentation, [S.I.], v. 9, n. 1, p. 48-57, 1953. Disponível em: <https://doi.org/10.1108/eb026190>. Acesso em: 03 dez. 2020.

WEINBERG, B. H. Indexing: history and theory. In: MCDONALD, JOHN, D.; LEVINE-CLARK, Michael (ed.). Encyclopedia of Library and Information Sciences. 4. ed. Boca Raton, FL: CRC Press, 2017. p. 1978-1991.

WELLISCH, H. H. Glossary of terminology in abstracting, classification, indexing, and thesaurus construction. Medford, New Jersey: Information Today, 2000. 77 p.

WELLISCH, H. H. The art of indexing and some fallacies of its automation. Logos, [S.I.], v. 3, n. 2, p. 69-76, 1992. Disponível em: <https://brill.com/view/journals/logo/3/2/article-p69_3. xml?language $=e n>$. Acesso em: 27 ago. 2020.

WILSON, P.Two kinds of power: an essay on bibliographical control. Berkeley: University of California Press. 1968. 